\title{
Thermodynamics of liquids: standard molar entropies and heat capacities of common solvents from 2PT molecular dynamics $\dagger$
}

\author{
Tod A. Pascal, ${ }^{a b}$ Shiang-Tai $\operatorname{Lin}^{c}$ and William A. Goddard III*ab \\ Received 19th August 2010, Accepted 7th October 2010 \\ DOI: $10.1039 / \mathrm{c0cp01549k}$
}

\begin{abstract}
We validate here the Two-Phase Thermodynamics (2PT) method for calculating the standard molar entropies and heat capacities of common liquids. In 2PT, the thermodynamics of the system is related to the total density of states (DoS), obtained from the Fourier Transform of the velocity autocorrelation function. For liquids this DoS is partitioned into a diffusional component modeled as diffusion of a hard sphere gas plus a solid component for which the $\operatorname{DoS}(v) \rightarrow 0$ as $v \rightarrow 0$ as for a Debye solid. Thermodynamic observables are obtained by integrating the DoS with the appropriate weighting functions. In the 2PT method, two parameters are extracted from the DoS self-consistently to describe diffusional contributions: the fraction of diffusional modes, $f$, and $\operatorname{DoS}(0)$. This allows $2 \mathrm{PT}$ to be applied consistently and without re-parameterization to simulations of arbitrary liquids. We find that the absolute entropy of the liquid can be determined accurately from a single short MD trajectory ( $20 \mathrm{ps}$ ) after the system is equilibrated, making it orders of magnitude more efficient than commonly used perturbation and umbrella sampling methods. Here, we present the predicted standard molar entropies for fifteen common solvents evaluated from molecular dynamics simulations using the AMBER, GAFF, OPLS AA/L and Dreiding II forcefields. Overall, we find that all forcefields lead to good agreement with experimental and previous theoretical values for the entropy and very good agreement in the heat capacities. These results validate $2 \mathrm{PT}$ as a robust and efficient method for evaluating the thermodynamics of liquid phase systems. Indeed $2 \mathrm{PT}$ might provide a practical scheme to improve the intermolecular terms in forcefields by comparing directly to thermodynamic properties.
\end{abstract}

\section{Introduction}

Modern quantum mechanics (QM) methods provide powerful means for predicting the energetics and enthalpies of molecules at low temperatures, including accurate estimates for solvation energies. ${ }^{1-3}$ However, neither QM nor molecular dynamics (MD) using forcefields (FF) have proved feasible for predicting accurate free energies from practical first principles calculations, primarily due to uncertainty in calculating entropy. Numerous methods have thus been proposed to calculate accurate entropies, although there is usually a tradeoff between accuracy and efficiency. Most perturbation MD methods ${ }^{4}$ based on Kirkwood-Zwanzig thermodynamic integration, ${ }^{5,6}$ have shown to be very accurate for a range of systems and can in principle lead to accurate free energy change from a reference system A to the target system B. However, complexities related to the choice of appropriate approximation formalism limit their straightforward application.

${ }^{a}$ Materials and Process Simulation Center, California Institute of Technology, Pasadena, CA 91125, USA.

E-mail:wag@wag.caltech.edu

${ }^{b}$ Graduate School of EEWS, Korea Advanced Institute of Science and Technology, Daejeon, Korea 305-701

${ }^{c}$ Department of Chemical Engineering, National Taiwan University, Taipei 10617, Taiwan

$\dagger$ Electronic supplementary information (ESI) available: Description of forcefield parameters, heats of vaporization, coefficient of thermal expansions and isothermal compressibilities. See DOI: 10.1039/ c0cp01549k
Alternatively, the free energy can be obtained from potential of mean-force simulations, ${ }^{7}$ which are markedly simpler, but not as accurate. Widom particle insertion ${ }^{8}$ schemes yield the chemical potential but require extensive sampling for all but the simplest of systems. Alternatively, Jorgensen and others have shown ${ }^{9-13}$ that Monte Carlo (MC) methods ${ }^{14}$ coupled with intermolecular forcefields can lead to accurate free energies of solution, but again this usually involves very long simulations to reduce the statistical uncertainty. Indeed, except in the context of thermodynamic integration using umbrella sampling, MD has not generally been useful for predicting the free energy or entropy of complex molecular systems.

It would be quite useful to have efficient ways to estimate the entropy directly from MD (thereby preserving the dynamical information lost from MC techniques). Indeed, entropy is expected to be the driving force behind most biochemical processes, ranging from protein folding and ligand/protein binding, ${ }^{15-17}$ to DNA transformations and recognition ${ }^{18}$ and hydrophobic effects. ${ }^{19,20}$ In particular, solubility and therefore miscibility of molecules in organic liquids may be dominated by changes in entropy, ${ }^{21-23}$ making accurate measures of the standard molar entropy critical to understanding solvation phenomena.

We propose here a practical approach to obtain accurate thermodynamics from short MD trajectories, which we validate by predicting entropies and specific heats of 15 standard 
solvents. Our approach is based on the $2 \mathrm{PT}$ method $^{24}$ for extracting absolute standard molar entropies and free energies from classical MD trajectories. In the 2PT paradigm, the entropy of the system is derived from the atomic velocity autocorrelation function $C(t)$ extracted from a 20 ps trajectory. The process is first to calculate the total density of states (DoS), from a Fast Fourier Transform of $C(t)$ to obtain $\operatorname{DoS}(v)$. Obtaining the thermodynamic properties from the $\operatorname{DoS}(v)$ of a liquid is complicated by diffusional effects (a finite DoS at $v=0$ ), which would lead to infinite values for the entropy for the standard harmonic oscillator partition function.

In the 2PT model, we assume that the DoS can be partitioned into a solid like component, $\operatorname{DoS}(v)_{\text {solid, }}$ and a diffusional component, $\operatorname{DoS}(v)_{\text {diff. This }} \operatorname{DoS}(v)_{\text {diff }}$ component is modeled in terms of a gas of hard spheres, completely described by $\operatorname{DoS}(v=0)$, the zero frequency intensity of the total DoS and $f$, the fraction of the $3 N$ degrees of freedom (dof) that are diffusional. These parameters $\operatorname{DoS}(v)$ and $f$ are extracted from the total DoS, leading to the vibrational $\operatorname{DoS}(v)_{\text {solid }}$ that can be analyzed using the standard harmonic oscillator partition function to account for the vibrational and librational components.

The original validation of the $2 \mathrm{PT}$ method was for a Lennard-Jones fluid, where very extensive MC calculations were available for the free energies for all regimes of the phase diagram, including solid, liquid, gas, supercritical, metastable, and unstable. Lin et al. ${ }^{24}$ showed that $2 \mathrm{PT}$ from short $10 \mathrm{ps}$ simulations gave excellent agreement with the MC for the entropies and free energies for all phases, including metastable and unstable regions.

The 2PT method has since been applied to several complex, condensed phase systems. For example, Lin et $a l .{ }^{25}$ showed that the water molecules in a full solvent simulation $(\sim 40000$ water molecules) of a generation 4 PAMAM Dendrimer (one molecule with 2244 atoms) leads to dramatically different entropies for the inner hydrophobic region, compared to the surface and bulk waters. Similarly, Jana et al. ${ }^{26}$ used 2PT to show that the entropies of water molecules in the grooves of DNA are significantly lower than in bulk water. More recently, Pascal et al. ${ }^{27}$ used $2 \mathrm{PT}$ to examine the entropic effects in binding a disaccharide (chitobiose) to the outer membrane protein A on Escherichia coli (a system involving 2589 atoms in the protein, 114 in the ligand and 42600 solvent/ lipid atoms). Here it was shown that various mutations led to a significant change in the contribution of entropy to the binding, so that enthalpies alone did not correlate well with experimental invasion rates, but that the $2 \mathrm{PT}$ derived free energies led to an excellent correlation. This work demonstrated the feasibility of calculating accurate ligand binding free energies and entropies on proteins embedded in a phospholipid membrane with explicit water molecules and salt.

While 2PT has been applied successfully to these and other complex systems, ${ }^{28,29}$ its performance in computing the absolute entropy and free energy of liquids other than water has not been demonstrated. In this paper, we use the 2PT method to calculate standard molar entropies and molar heat capacities of 15 common organic liquids. Of course the 2PT analysis cannot be better than the forcefield used, so we test the accuracy of several forcefields: AMBER-2003, ${ }^{30,31}$ General
Amber Force Field (GAFF), ${ }^{32}$ OPLS AA/L $\mathrm{L}^{11,33}$ and Dreiding II. $^{34}$ Overall, we find good agreement with experiment from all these forcefields, but OPLS AA/L, derived to fit MC simulations of liquids, was the best. Perhaps more importantly, we find that after equilibration 20 ps of MD leads to deviations in the standard molar entropy of $0.25 \mathrm{cal} \mathrm{mol}^{-1} \mathrm{~K}^{-1}$ (or $0.6 \%$ ).

In the 2PT framework, the heat capacity is calculated (as any other thermodynamic quantity) directly from the DoS by applying the appropriate weighting function. In this paper we show that the calculated molar heat capacities are in very good agreement with experiment and in excellent agreement with other theoretical methods, further validating the 2PT method.

Finally, we present the partition of the total entropy of these solvents into the rotational, translational and internal vibrational components. We find that $46 \%$ of the entropy of the liquids arises from translation, $37 \%$ from rotation and $17 \%$ from intra-molecular vibrations, with large variations depending on the nature of the liquid. This is the first time that such a theoretical component analysis has been reported for these organic liquids. We expect that such analyses may be useful in formulating macroscale methods of estimating entropies of solvation.

Taken together, we demonstrate that the 2PT method can be applied without reparameterization to study liquids, producing precise results from standard molecular dynamics forcefields and simulation codes. The remainder of the paper is organized as follows: Section II presents the major results with discussions. Section III presents the theoretical background of the 2PT method and details for the application to the systems considered here.

\section{Results and discussion}

\section{II.a Applicability of forcefields for liquid simulations}

Table 1 compares the computed static dielectric constants (see Appendix A.II.3 of ESI $\dagger$ ) of the 15 liquids in this study. We find that all forcefields (FF) underestimate the dielectric constants, with the magnitude of the error depending on the nature of the solvent. This is expected since these FF all use fixed charges and hence cannot account for the molecular polarizability contribution to the dielectric constant. We plan later to use the QEq method ${ }^{35}$ to include such polarization effects.

For non-polar solvents, the OPLS AA/L FF has the best performance, with a mean absolute deviation-MAD-error of $0.078(2.5 \%)$ and a root mean squared-RMS-error (a measurement of the variance) of 0.103 . The next best is GAFF (3.6\% error), the AMBER 2003 FF (15.5\%) and finally the Dreiding FF $(20 \%)$. The excellent performance of OPLS AA/L might be expected since the charges of benzene, ${ }^{10}$ chloroform, ${ }^{10}$ furan ${ }^{36}$ and toluene ${ }^{10}$ were explicitly parameterized to reproduce the experimental dielectric properties.

The gas-phase RESP $^{37}$ charges in GAFF generally are more polar than the solution phase fitted charges in OPLS (Table S1, ESI $\dagger$ ). Perhaps unsurprisingly, the calculated gas phase dipole moments (Table 2) are closer to the experimental 
Table 1 Comparison of the calculated static dielectric constants $\left(\varepsilon_{0}\right)$ of all 15 organic liquids to experiments. Effects due to charge polarization are not included

\begin{tabular}{|c|c|c|c|c|c|c|c|c|c|}
\hline \multirow[b]{2}{*}{ Non-polar } & \multirow[t]{2}{*}{$\operatorname{Exp}^{a}$} & \multicolumn{2}{|c|}{ AMBER $2003^{b, c, d}$} & \multicolumn{2}{|c|}{ Dreiding $^{c, e}$} & \multicolumn{2}{|c|}{$\mathrm{GAFF}^{b, c, d}$} & \multicolumn{2}{|c|}{ OPLS AA/ $\mathrm{L}^{f}$} \\
\hline & & & & & & & & & \\
\hline Benzene & 2.283 & 1.031 & 0.000 & 1.018 & 0.000 & 1.014 & 0.000 & 1.027 & 0.000 \\
\hline Chloroform & 4.807 & 2.136 & 0.000 & 1.738 & 0.000 & 3.236 & 0.001 & 3.636 & 0.001 \\
\hline 1,4-Dioxane & 2.219 & 1.082 & 0.000 & 1.079 & 0.000 & 1.055 & 0.000 & 1.057 & 0.000 \\
\hline furan & 2.940 & 1.232 & 0.001 & 1.160 & 0.000 & 1.668 & 0.000 & 1.534 & 0.000 \\
\hline Toluene & 2.379 & 1.049 & 0.000 & 1.182 & 0.000 & 1.039 & 0.000 & 1.204 & 0.000 \\
\hline M.A.D. & - & 0.456 & - & 0.587 & - & 0.106 & - & 0.078 & 0.456 \\
\hline RMS error & - & 0.626 & - & 0.812 & - & 0.152 & - & 0.103 & 0.626 \\
\hline \multicolumn{10}{|l|}{ Polar-aprotic } \\
\hline Acetonitrile & 36.640 & 18.843 & 0.126 & 17.908 & 0.113 & 22.429 & 0.186 & 14.468 & 0.069 \\
\hline Acetone & 21.100 & 12.319 & 0.047 & 19.535 & 0.138 & 12.343 & 0.048 & 14.417 & 0.069 \\
\hline DMSO & 47.240 & 53.590 & 1.205 & 45.723 & 0.865 & 59.147 & 1.500 & 49.221 & 0.975 \\
\hline THF & 7.520 & 10.263 & 0.030 & 16.701 & 0.095 & 9.487 & 0.023 & 5.340 & 0.005 \\
\hline M.A.D. & - & 8.918 & - & 7.787 & - & 9.211 & - & 7.454 & 8.918 \\
\hline RMS error & - & 11.034 & - & 11.547 & - & 11.599 & - & 10.550 & 11.034 \\
\hline \multicolumn{10}{|l|}{ Polar protic } \\
\hline Acetic acid & 6.200 & - & - & 4.623 & 0.001 & - & - & 4.311 & 0.002 \\
\hline Ethanol & 25.300 & 16.020 & 0.083 & 7.796 & 0.015 & 16.184 & 0.072 & 19.075 & 0.120 \\
\hline Ethylene glycol & 41.400 & 37.212 & 0.514 & 13.284 & 0.042 & 11.708 & 0.013 & 30.389 & 0.350 \\
\hline Methanol & 33.000 & 24.678 & 0.228 & 14.965 & 0.074 & 33.399 & 0.423 & 25.540 & 0.249 \\
\hline NMA & 179.000 & 85.662 & 0.836 & 69.445 & 1.794 & 99.101 & 1.748 & 62.611 & 1.599 \\
\hline TFE & 27.680 & 13.437 & 0.054 & 33.408 & 0.422 & 14.294 & 0.064 & 23.931 & 0.201 \\
\hline M.A.D. & - & 26.986 & - & 27.126 & - & 22.765 & - & 30.645 & 26.986 \\
\hline RMS error & - & 37.883 & - & 41.704 & - & 31.856 & - & 45.148 & 37.883 \\
\hline
\end{tabular}

Table 2 Comparison of the calculated gas-phase dipole moments (Debye) vs. experiment. Each liquid is first minimized in the appropriate forcefield

\begin{tabular}{llllll}
\hline & $\operatorname{Exp}^{a}$ & AMBER 03 & Dreiding & GAFF & OPLS AA/L \\
\hline Acetic acid & 1.70 & 1.678 & 2.307 & 1.506 & 1.542 \\
Acetone & 2.88 & 3.226 & 3.608 & 3.194 & 3.111 \\
Acetonitrile & 3.92 & 4.035 & 4.681 & 4.030 & 4.129 \\
Benzene & 0.00 & 0.000 & 0.000 & 0.000 & 0.000 \\
Chloroform & 1.04 & 1.085 & 0.934 & 1.083 & 1.462 \\
1,4-Dioxane & 0.45 & 0.000 & 0.000 & 0.000 & 0.000 \\
DMSO & 3.96 & 4.933 & 5.450 & 4.711 & 4.700 \\
Ethanol & 1.69 & 1.876 & 1.921 & 1.900 & 2.323 \\
Ethylene glycol & - & 0.000 & 0.000 & 0.000 & 0.000 \\
Furan & - & 0.868 & 0.429 & 0.843 & 0.732 \\
Methanol & 1.70 & 2.150 & 2.060 & 2.177 & 2.274 \\
NMA & 4.04 & 4.414 & 4.881 & 4.378 & 4.030 \\
THF & 1.75 & 2.621 & 3.617 & 2.690 & 1.972 \\
Toluene & 0.36 & 0.211 & 0.606 & 0.221 & 0.566 \\
TFE & - & 3.563 & 4.640 & 3.624 & 4.087 \\
${ }^{a}$ Ref. 82. & & & & & \\
\hline
\end{tabular}

gas phase values. This relatively good performance of GAFF indicates that these gas phase static charges are transferable for simulations of non-polar liquids, greatly simplifying FF development. The relatively poor performance of the AMBER 2003 FF compared to the GAFF is also not surprising, since the AMBER forcefield was not optimized for simulations of liquids. We find that the Mulliken charges used with the generic Dreiding FF are more polar than the RESP charges of GAFF, leading Dreiding to overestimate the gas phase dipole moments, relative to GAFF and charges that are not transferable to the condensed phase.
The agreement with experimental dielectric constants deteriorates considerably for polar solvents. We find MAD errors of $8.92,7.79,9.21$ and $7.5(33 \%, 28 \%, 32 \%$ and $27 \%)$ for the aprotic solvents for the AMBER, Dreiding, GAFF and OPLS, respectively, and significantly worse agreement for the protic solvents, with errors greater than $50 \%$ for all but the GAFF forcefield $(44 \%)$. The largest errors are observed for NMA (the most polar solvent), where the dielectric constant is underestimated by $93.4,109.6,80.0$ and 116.4 respectively ( $c f$. the experimental value of 179.0). We again attribute these large errors to the lack of charge polarization in these forcefields. Indeed Anisimov et al. ${ }^{38}$ showed that the dielectric constants of common alcohols are underestimated by $36 \%$ using non-polorizable forcefields compared to polarizable forcefields, and that polarizable forcefields show significantly better agreement to experiments.

The AMBER, GAFF and OPLS forcefields slightly underestimate the liquid densities and molar volumes, with average errors of $-1.4 \%,-2.2 \%$ and $-1.6 \%$, respectively, across all liquids (Table 3 ). Since the density of the liquid is a parameter used in fitting these forcefields, the better performance compared to the dielectric constant (usually not a fitting parameter except the cases of OPLS outlined above) is to be expected. This indicates that the vdW parameters, and specifically the equilibrium distance $R_{0}$, are tuned to compensate for inaccuracies in the electrostatics. The Dreiding underestimates the densities by $10 \%$, which might be expected due to the generic nature of this FF. The results obtained here could be used to optimize the Dreiding vdW parameters. 
Table 3 Comparison of experimental and predicted densities and molar volumes of organic liquids. Calculated values obtained from statistical averaging over $2.5 \mathrm{~ns} \mathrm{MD}$, sampled every $100 \mathrm{ps}$. Numbers in parentheses indicate the uncertainty ${ }^{a}$

\begin{tabular}{|c|c|c|c|c|c|c|c|c|c|c|c|c|c|c|}
\hline \multirow[b]{3}{*}{ Acetic acid } & \multicolumn{9}{|c|}{ Density $\langle\rho\rangle / \mathrm{g} \mathrm{cm}^{-3}$} & \multicolumn{5}{|c|}{ Molar volume $^{b}\langle V\rangle / \mathrm{cm}^{3}$} \\
\hline & \multirow{2}{*}{$\frac{\operatorname{Exp}^{c}}{1.045}$} & \multicolumn{2}{|c|}{ AMBER 2003} & \multicolumn{2}{|c|}{ Dreiding } & \multicolumn{2}{|c|}{ GAFF } & \multicolumn{2}{|c|}{ OPLS AA/L } & \multirow{2}{*}{$\frac{\operatorname{Exp}^{c}}{95.45}$} & \multirow[t]{2}{*}{ AMBER 2003} & \multirow{2}{*}{$\frac{\text { Dreiding }}{97.75}$} & \multirow[t]{2}{*}{ GAFF } & \multirow{2}{*}{$\frac{\text { OPLS AA } / \mathrm{L}}{95.19}$} \\
\hline & & & & 1.020 & (99) & & & 1.047 & (16) & & & & & \\
\hline Acetone & 0.785 & 0.757 & (19) & 0.697 & (32) & 0.764 & $(31)$ & 0.777 & (26) & 122.9 & 127.33 & 138.41 & 126.25 & 124.11 \\
\hline Acetonitrile & 0.786 & 0.729 & (18) & 0.730 & (18) & 0.705 & (27) & 0.711 & (71) & 86.75 & 93.53 & 93.37 & 96.62 & 95.92 \\
\hline Benzene & 0.877 & 0.827 & (31) & 0.802 & (54) & 0.796 & (62) & 0.913 & (26) & 147.96 & 156.82 & 161.77 & 162.90 & 141.98 \\
\hline Chloroform & 1.479 & 1.380 & (34) & 1.331 & (55) & 1.412 & (34) & 1.447 & (82) & 134.03 & 143.59 & 148.96 & 140.40 & 136.96 \\
\hline 1,4-Dioxane & 1.034 & 1.088 & (26) & 0.871 & (27) & 1.068 & (37) & 0.987 & (24) & 141.51 & 134.49 & 167.89 & 137.03 & 148.15 \\
\hline DMSO & 1.101 & 1.096 & (28) & 1.089 & (15) & 1.103 & (35) & 1.095 & (23) & 117.82 & 118.38 & 119.09 & 117.59 & 118.42 \\
\hline Ethanol & 0.789 & 0.790 & (27) & 0.642 & (34) & 0.785 & (33) & 0.783 & (18) & 96.90 & 96.83 & 119.11 & 97.38 & 97.66 \\
\hline Ethylene glycol & 1.114 & 1.166 & (12) & 0.997 & (44) & 1.115 & $(50)$ & 1.064 & (24) & 92.55 & 88.38 & 103.31 & 92.38 & 96.83 \\
\hline Furan & 0.951 & 0.959 & (29) & 0.852 & (32) & 0.950 & (32) & 0.972 & (37) & 118.80 & 117.86 & 132.64 & 118.96 & 116.25 \\
\hline Methanol & 0.791 & 0.801 & (27) & 0.644 & (29) & 0.799 & (27) & 0.766 & (24) & 67.22 & 66.42 & 82.62 & 66.57 & 69.49 \\
\hline NMA & 0.937 & 0.954 & (18) & 0.847 & (24) & 0.952 & (21) & 0.952 & (20) & 129.50 & 127.14 & 143.32 & 127.47 & 127.54 \\
\hline THF & 0.883 & 0.909 & (32) & 0.826 & (19) & 0.901 & (43) & 0.786 & (24) & 135.53 & 131.68 & 144.98 & 132.84 & 152.25 \\
\hline Toluene & 0.862 & 0.816 & (23) & 0.782 & (10) & 0.822 & (21) & 0.900 & (33) & 177.41 & 187.47 & 195.57 & 186.09 & 170.06 \\
\hline TFE & 1.384 & & & 1.300 & (20) & & & 1.354 & $(52)$ & 119.99 & & 127.79 & & 122.68 \\
\hline
\end{tabular}

\section{II.b Convergence, efficiency and precision of the 2PT method}

Lin et $a l .{ }^{24}$ showed that the entropies predicted with $2 \mathrm{PT}$ for a LJ gas converge for just 10 ps of dynamics. To validate the time needed for convergence, we calculated the properties of benzene using OPLS AA/L for 5 independent 1 ns trajectories, calculating the properties for various length trajectories: $1 \mathrm{ps}$, 4 ps, 10 ps, 20 ps, 40 ps, 100 ps and 200 ps (Fig. 3). We find that by 20 ps the entropy and heat capacity are converged, while the self-diffusivity took 50 to 100 ps to converge (Fig. S1, ESI $\dagger$ ). This convergence in the thermodynamic quantities is consistent with a recent study of Lin et al. ${ }^{39}$ that found that the entropy of liquid water converges after 10 to $50 \mathrm{ps}$.

Due to the short 20 ps trajectories required and the efficiency of FFTs, the 2PT calculations presented here require only a trivial increase in additional computation time. This allows one to calculate the system thermodynamics on-the-fly during dynamics. Such calculations provide a rigorous check of numerical stability and precision of the method.

Fig. 4 reports the standard molar entropies and heat capacities for acetic acid, benzene and DMSO with OPLS $\mathrm{AA} / \mathrm{L}$. Here, we used the last 20 ps of dynamics to evaluate the thermodynamic properties every 100 ps during the $2.5 \mathrm{~ns}$ dynamics, for a total of 25 data points. Convergence is observed after only $300 \mathrm{ps}$ of equilibration, with fluctuations of $0.36 \mathrm{cal} \mathrm{mol}^{-1} \mathrm{~K}^{-1}$ in specific heat $(0.6 \%)$. This indicates that $2 \mathrm{PT}$ gives robust and precise thermodynamic quantities from short MD trajectories. The additional simulation and computational time is also minimal, with the trajectories generated automatically during regular dynamics and the post trajectory analysis taking less than $2 \%$ of the total simulation time. For example, the total time to simulate 512 molecules of benzene for $2.5 \mathrm{~ns}$ with LAMMPS took approximately $110 \mathrm{CPU}$ hours on a $3.2 \mathrm{GHz}$ Intel Xenon processor, while the additional analysis of the 25 NVT trajectories to obtain the 2PT prediction took an additional 16 minutes $(0.2 \%)$. Further, the average values of the entropy and heat capacity calculated every 500 ps ( 5 trajectories) are within $0.1 \%$ of the average calculated every 100 ps, showing that accurate thermodynamics can be obtained from uncorrelated or correlated trajectories.

In all our simulations, we chose not to constrain the motion of the hydrogen atoms by the $\mathrm{SHAKE}^{40}$ algorithm, as is commonly the practice in the AMBER/GAFF and OPLS forcefields. While these constraints would presumably not affect the dynamics, ${ }^{41}$ the calculated thermodynamics depends on integrating over the entire DoS, thus SHAKE might affect the thermodynamics. Conversely, the high frequency of the vibrations may render any effect due to SHAKE minimal, as high frequency modes contribute exponentially less to the thermodynamics than low frequency modes. We note however that the 2PT formalism allows for accurate calculation of thermodynamic quantities regardless of external constraints, by accounting for the removed degrees of freedom (Dof): the Dof is used to calculate the system's temperature from the atomic velocities.

\section{II.c Comparison of standard molar entropies vs. experiment}

Fig. 5 and Table 4 present the standard molar entropies $S^{0}$. Contributions due to configurational entropy are included by statistical averaging over 5 discrete and uncorrelated microstates, obtained from 20 ps trajectories every 500 ps of the 2.5 ns dynamics, as described previously. Effects due to configurational changes are captured in the diffusive component and explicitly included in our model.

All FF underestimate $S^{0}$, with average errors of -4.13 , $-2.12,-6.36,-2.97 \mathrm{cal} \mathrm{mol}^{-1} \mathrm{~K}^{-1}$ for AMBER 2003, Dreiding, Gaff and OPLS AA/L, respectively, or approximately 5 to $15 \%$. As was the case with the dielectric constant, the largest discrepancy occurs in the polar solvents, in particular ethylene glycol (average of $16 \%$ error) and DMSO (average of $15 \%$ error). This may again point to the deficiency of using a fixed charge model, since the diffusion constant of other liquids ${ }^{42}$ is known to be also affected by the lack of polarization. Selfdiffusion and other low frequency librational modes contribute 
Table 4 Comparison of average standard molar entropy $S^{0}\left(\mathrm{cal} \mathrm{mol}^{-1} \mathrm{~K}^{-1}\right)$ for the 15 liquids and 4 different forcefields in this study. Entropies evaluated last $20 \mathrm{ps}$ every $500 \mathrm{ps}$ of $2.5 \mathrm{~ns}$ MD simulation. Average fluctuations of $0.31 \mathrm{kcal} \mathrm{mol}^{-1}$ molecule $^{-1}$ is observed over all forcefields. Overall, the OPLS AA/L forcefield is the best performer, with a mean absolute error (M.A.D) of $1.47 \mathrm{cal} \mathrm{mol}^{-1} \mathrm{molecule}^{-1}$, an average error of $-5.85 \mathrm{cal} \mathrm{mol}^{-1}$ molecule $^{-1}$ and a $R^{2}$ correlation coefficient of $92 \%$

\begin{tabular}{|c|c|c|c|c|c|c|c|c|c|c|}
\hline \multirow[b]{2}{*}{ Solvent } & \multirow[b]{2}{*}{$\operatorname{Exp}^{a}$} & \multirow[b]{2}{*}{ Best estimate $^{c}$} & \multicolumn{2}{|c|}{ AMBER 03} & \multicolumn{2}{|c|}{ Dreiding } & \multicolumn{2}{|l|}{ GAFF } & \multicolumn{2}{|c|}{ OPLS AA/L } \\
\hline & & & Avg & \pm & Avg & \pm & Avg & \pm & Avg & \pm \\
\hline Acetic acid & 37.76 & & 32.23 & 0.25 & 37.79 & 2.35 & 30.67 & 0.17 & 35.13 & 0.22 \\
\hline Acetone & 47.90 & & 44.72 & 0.15 & 44.26 & 0.26 & 44.79 & 0.21 & 47.27 & 0.08 \\
\hline Acetonitrile & 35.76 & & 38.06 & 0.15 & 34.08 & 0.21 & 34.75 & 0.28 & 33.99 & 0.22 \\
\hline Benzene & 41.41 & & 40.58 & 0.22 & 39.01 & 0.25 & 38.37 & 0.52 & 41.19 & 0.16 \\
\hline 1,4-Dioxane & 46.99 & & 39.47 & 0.22 & 41.71 & 0.27 & 38.00 & 0.20 & 42.82 & 0.26 \\
\hline DMSO & 45.12 & & 39.57 & 0.25 & 38.71 & 0.13 & 37.99 & 0.24 & 39.10 & 0.12 \\
\hline Ethanol & 38.21 & & 33.92 & 0.33 & 41.97 & 0.13 & 30.36 & 0.13 & 33.63 & 0.16 \\
\hline Ethylene glycol & 39.89 & & 30.43 & 0.05 & 40.13 & 0.31 & 28.95 & 0.18 & 33.62 & 0.16 \\
\hline Furan & 42.22 & & 39.09 & 0.00 & 38.88 & 0.38 & 37.60 & 0.25 & 40.03 & 0.23 \\
\hline Methanol & 30.40 & & 28.61 & 0.10 & 25.87 & 0.26 & 26.13 & 0.12 & 29.12 & 0.08 \\
\hline THF & 48.71 & & 41.89 & 0.22 & 48.45 & 0.12 & 38.01 & 0.18 & 46.96 & 0.28 \\
\hline Toluene & 52.81 & & 48.98 & 0.23 & 45.91 & 0.24 & 45.30 & 0.19 & 48.67 & 0.23 \\
\hline M.A.D. ${ }^{b}$ & & & 2.39 & & 2.48 & & 2.62 & & 1.72 & \\
\hline Avg. error & & & -4.13 & & -2.53 & & -6.36 & & -2.97 & \\
\hline RMS error & & & 3.17 & & 3.12 & & 3.15 & & 2.03 & \\
\hline$R^{2}$ & & & 0.75 & & 0.74 & & 0.76 & & 0.90 & \\
\hline Chloroform & & 43.01 & 47.47 & 0.10 & 47.44 & 0.30 & 53.88 & 0.22 & 45.98 & 0.31 \\
\hline NMA & & 40.23 & 41.86 & 0.29 & 33.51 & 0.24 & 40.18 & 0.14 & 43.20 & 0.07 \\
\hline TFE & & 43.54 & 48.54 & 0.30 & 46.07 & 0.08 & 44.30 & 0.23 & 46.51 & 0.22 \\
\hline
\end{tabular}

${ }^{a}$ NIST Reference Database Number $69 .{ }^{64 b}$ Mean absolute deviation. ${ }^{c}$ No experimental values are available for chloroform, NMA and TFE. We estimate their values here but subtracting the average error from the calculated OPLS AA/L values.

most to the entropy calculated from approaches that rely on the DoS such as 2PT.

While none of the forcefields reproduce the experimental $S^{0}$, the OPLS forcefield shows the best overall correlation with experiment, with a $1.72 \mathrm{cal} \mathrm{mol}^{-1} \mathrm{~K}^{-1} \mathrm{MAD}$ and a $90 \%$ correlation. Particularly exciting here is the performance of the Dreiding forcefield $\left(2.5 \mathrm{cal} \mathrm{mol}^{-1} \mathrm{~K}^{-1}\right.$ MAD and $74 \%$ correlation), since no parameters related to the thermodynamics of liquids were used in determining the forcefield parameters. The AMBER and GAFF forcefields have performance similar to Dreiding: 2.39 and $2.67 \mathrm{cal} \mathrm{mol}^{-1} \mathrm{~K}^{-1} \mathrm{MAD}$ and $75 \%$ and $76 \%$ correlation respectively. We find that $2 \mathrm{PT}$ predicts standard molar entropies of these pure liquids to within $0.25 \mathrm{cal} \mathrm{mol}^{-1} \mathrm{~K}^{-1}(0.6 \%)$ standard deviation over all forcefields.

Since there are no experimental standard molar entropy values for chloroform, NMA and TFE, we provide here a priori predictions based on the OPLS AA/L forcefield average error of $-2.97 \mathrm{cal} \mathrm{mol}^{-1} \mathrm{~K}^{-1}$ : 43.01 for chloroform, 40.23 for NMA and $43.54 \mathrm{cal} \mathrm{mol}^{-1} \mathrm{~K}^{-1}$ for TFE.

\section{II.d Comparison of molar heat capacities vs. experiment}

In 2PT we prefer to keep the volume constant (NVT MD) leading to $C_{v}$ and Helmholtz free energies, because we consider this to be the least ambiguous framework for describing the DoS. However experiments are generally carried out under conditions of NPT, leading to $C_{p}$ and Gibbs free energies. To compare the $C_{v}$ from $2 \mathrm{PT}$ to the $C_{p}$ from experiment, we apply a correction:

$$
\begin{aligned}
C_{p} & =\left(\frac{\partial H}{\partial T}\right)_{p}=C_{v}+\Delta C_{v, p} \\
& =C_{v}+T\left(\frac{\partial p}{\partial T}\right)_{N, V}\left(\frac{\partial V}{\partial T}\right)_{N, P}=C_{v}+V T \frac{\alpha_{p}^{2}}{\kappa_{T}}
\end{aligned}
$$

where $\alpha_{p}$ is the coefficient of thermal expansion (Table S2, ESI $\dagger$ ) and $\kappa_{T}$ is the isothermal compressibility (Table S3, ESI $\dagger$ ). We find that the corrections to the $C_{v}$ are all less than $0.25 \mathrm{cal} \mathrm{mol}^{-1} \mathrm{~K}^{-1}$ (Table S4, ESI $\dagger$ ) (Fig. 6).

Overall, all forcefields reproduce the experimental heat capacities to within 5\%. More importantly, the values calculated using the $2 \mathrm{PT}$ approach show a $96 \%$ correlation to the approach used by Jorgensen and coworkers ${ }^{9,10,12,13,36,43}$ with the OPLS forcefield, which was based on extensive Monte Carlo sampling. This validates that 2PT can capture the essential physics in these systems from short 20 ps MD trajectories. Further, the statistical deviations in our calculated heat capacities are $0.2 \mathrm{cal} \mathrm{mol}^{-1} \mathrm{~K}^{-1}$, or $0.5 \%$ (Table 5).

\section{II.e Components of liquid entropy}

An attractive feature of $2 \mathrm{PT}$ is the facility to separate the individual components of the entropy as detailed in Section III.c.ii. We performed this decomposition for all the liquids, with the OPLS AA/L forcefield (Table 6). Here we find the ratio of the contributions to the entropy of $2: 4: 5$ for valence vibrations : rotation : translation across all molecules, leading to a non-negligible contribution of $17 \%$ to the entropy from the internal vibrations. As expected, the vibrational entropy is greatest for the large flexible solvents $(31 \%$, $24 \%$ and $21 \%$ for NMA, TFE and ethylene glycol respectively) and least for the small rigid solvents $3 \%$ and $6 \%$ for acetonitrile and methanol respectively). Since the vibrational component of the total DoS is analogous to the experimental IR and Raman spectra, forcefields that more closely reproduce the experimental vibrational frequencies should lead to improved entropies. For illustrative purposes, we show the vibrational DoS for chloroform using the OPLS AA/L forcefield in Fig. 2b. The vibrational frequencies are on average 
Table 5 Comparison of the calculated constant pressure heat capacity $C_{\mathrm{p}}\left(\mathrm{cal} \mathrm{mol}^{-1} \mathrm{~K}^{-1}\right)^{a}$ with experiment. Here, the Dreiding forcefield has a similar M.A.D. $\left(2.02 \mathrm{cal} \mathrm{mol}^{-1} \mathrm{~K}^{-1}\right)$ to the OPLS AA/L forcefield $\left(2.00 \mathrm{cal} \mathrm{mol}^{-1} \mathrm{~K}^{-1}\right)$, although the OPLS forcefield has a smaller average error $\left(-0.9 \mathrm{cal} \mathrm{mol}^{-1} \mathrm{~K}^{-1}\right.$ vs. $\left.-3.05 \mathrm{cal} \mathrm{mol}^{-1} \mathrm{~K}^{-1}\right)$ due to cancelling of errors

\begin{tabular}{|c|c|c|c|c|c|c|c|c|c|c|c|}
\hline & \multirow[b]{2}{*}{$\operatorname{Exp}^{b}$} & \multirow[b]{2}{*}{ Best Estimate } & \multicolumn{2}{|c|}{ AMBER 2003} & \multicolumn{2}{|c|}{ Dreiding } & \multicolumn{2}{|l|}{ GAFF } & \multicolumn{2}{|c|}{ OPLS AA/L } & \multirow{2}{*}{$\begin{array}{l}\text { Other calculated } \\
\text { values }\end{array}$} \\
\hline & & & Avg & \pm & Avg & \pm & Avg & \pm & Avg & \pm & \\
\hline Acetic acid & 29.42 & & & & 26.44 & 0.76 & & & 26.76 & 0.12 & $\begin{array}{l}26.06^{9} \\
29.4^{84} \\
30.6^{43}\end{array}$ \\
\hline Acetone & 29.98 & & 29.37 & 0.10 & 28.29 & 0.12 & 29.31 & 0.14 & 30.88 & 0.13 & $30.2^{84}$ \\
\hline Acetonitrile & 21.91 & & 23.38 & 0.07 & 22.90 & 0.10 & 20.75 & 0.23 & 19.45 & 0.09 & $19.43^{12}$ \\
\hline Benzene & 32.43 & & 29.35 & 0.21 & 26.73 & 0.13 & 23.52 & 0.25 & 30.73 & 0.17 & $\begin{array}{l}31.2^{10} \\
31.8^{84}\end{array}$ \\
\hline Chloroform & 27.31 & & 26.10 & 0.18 & 25.16 & 0.17 & 30.23 & 0.09 & 25.18 & 0.17 & - \\
\hline 1,4-Dioxane & 35.77 & & 34.32 & 0.13 & 33.00 & 0.07 & 33.15 & 0.27 & 36.74 & 0.18 & $36.0^{85}$ \\
\hline DMSO & 35.71 & & 31.39 & 0.17 & 30.80 & 0.14 & 30.64 & 0.13 & 32.15 & 0.14 & $\begin{array}{l}36.0^{86} \\
34.75^{87}\end{array}$ \\
\hline Ethanol & 26.86 & & 24.82 & 0.26 & 24.41 & 0.13 & 23.46 & 0.11 & 26.03 & 0.12 & $\begin{array}{l}26.1^{13} \\
23.9^{88}\end{array}$ \\
\hline $\begin{array}{l}\text { Ethylene } \\
\text { glycol }\end{array}$ & 35.80 & & 28.39 & 0.09 & 27.88 & 0.15 & 27.38 & 0.20 & 29.98 & 0.15 & - \\
\hline Furan & 27.38 & & 24.91 & 0.10 & 23.24 & 0.14 & 22.07 & 0.16 & 26.22 & 0.08 & $26.68^{36}$ \\
\hline Methanol & 19.00 & & 18.69 & 0.19 & 18.46 & 0.16 & 18.07 & 0.17 & 19.37 & 0.09 & $\begin{array}{l}20.0^{13} \\
26.0^{43} \\
22.5^{88}\end{array}$ \\
\hline THF & 29.66 & & 30.81 & 0.14 & 28.91 & 0.12 & 29.31 & 0.09 & 33.33 & 0.20 & $31.9^{43}$ \\
\hline Toluene & 37.55 & & 37.40 & 0.15 & 33.87 & 0.10 & 31.19 & 0.11 & 39.08 & 0.14 & - \\
\hline M.A.D. & & & 1.93 & & 2.02 & & 2.62 & & 2.00 & & \\
\hline Avg. error & & & -1.75 & & -3.05 & & -3.93 & & -0.90 & & \\
\hline RMS error & & & 3.01 & & 3.84 & & 4.92 & & 2.62 & & \\
\hline$R^{2}$ & & & 0.79 & & 0.78 & & 0.70 & & 0.83 & & \\
\hline NMA & & 36.60 & 34.49 & 0.12 & 32.76 & 0.13 & 33.10 & 0.09 & 35.70 & 0.07 & $39.7^{43,63}$ \\
\hline TFE & & 36.46 & 35.39 & 0.16 & 35.23 & 0.09 & 34.54 & 0.10 & 35.56 & 0.14 & - \\
\hline
\end{tabular}

${ }^{a} C_{p}$ is obtained from the calculated $C_{v}$ by eqn (1). The corrections to the heat capacity $\Delta C_{v}$ are all $<0.25 \mathrm{cal} \mathrm{mol}^{-1} \mathrm{~K}^{-1}$ (Table S4, ESI $\dagger$ ). ${ }^{b} \mathrm{NIST}$ Reference Database Number 69. ${ }^{64}$

Table 6 Self-diffusion constant $D\left(\mathrm{~cm}^{2} \mathrm{~s}^{-1}\right)$, vibrational $\left(S_{\mathrm{vib}}\right)$, rotational $\left(S_{\mathrm{rot}}\right)$, and translational $\left(S_{\text {trans }}\right)$ components of $S^{0}\left(\mathrm{cal} \mathrm{mol}^{-1} \mathrm{~K}^{-1}\right)$ and the 2PT fluidicity parameters for all 15 liquids in this study, calculated with the OPLS AA/L forcefield. Results for the F3C, SPC/E and TIP4P-Ew water models are included for comparative purposes

\begin{tabular}{|c|c|c|c|c|c|c|c|c|c|c|c|}
\hline & \multicolumn{6}{|c|}{ Standard molar entropy $S^{0} / \mathrm{cal} \mathrm{mol}^{-1} \mathrm{~K}^{-1}$} & \multicolumn{2}{|c|}{ Fluidicity factor } & \multicolumn{3}{|c|}{$D \times 10^{-5} / \mathrm{cm}^{2} \mathrm{~s}^{-1}$} \\
\hline & \multicolumn{2}{|l|}{$\underline{S_{\mathrm{vib}}}$} & \multicolumn{2}{|l|}{$\underline{S_{\mathrm{rot}}}$} & \multicolumn{2}{|l|}{$S_{\text {trans }}$} & \multirow{2}{*}{$f_{\text {trans }}$} & \multirow{2}{*}{$f_{\text {rot }}$} & \multirow{2}{*}{$\operatorname{MSD}^{a}$} & \multirow{2}{*}{$\mathrm{GK}^{b}$} & \multirow{2}{*}{$\operatorname{Exp}^{c}$} \\
\hline & Avg & \pm & Avg & \pm & Avg & \pm & & & & & \\
\hline Acetic acid & 6.28 & 0.06 & 13.38 & 0.08 & 15.48 & 0.10 & 0.16 & 0.12 & 1.03 & 1.18 & \\
\hline Acetone & 11.08 & 0.04 & 16.84 & 0.04 & 19.35 & 0.05 & 0.34 & 0.29 & 4.39 & 5.09 & \\
\hline Acetonitrile & 0.93 & 0.02 & 13.86 & 0.08 & 19.20 & 0.14 & 0.40 & 0.30 & 7.25 & 7.93 & \\
\hline Benzene & 4.74 & 0.06 & 16.63 & 0.06 & 19.83 & 0.09 & 0.30 & 0.29 & 3.45 & 3.77 & \\
\hline Chloroform & 5.65 & 0.02 & 19.20 & 0.15 & 21.12 & 0.16 & 0.33 & 0.30 & 3.22 & 3.76 & \\
\hline 1,4-Dioxane & 8.43 & 0.05 & 16.43 & 0.09 & 17.97 & 0.15 & 0.20 & 0.20 & 1.69 & 1.82 & \\
\hline DMSO & 8.87 & 0.06 & 13.93 & 0.08 & 16.31 & 0.10 & 0.16 & 0.13 & 0.63 & 1.09 & \\
\hline Ethanol & 4.51 & 0.01 & 13.16 & 0.06 & 15.95 & 0.09 & 0.20 & 0.15 & 1.54 & 1.82 & \\
\hline Ethylene glycol & 6.94 & 0.02 & 12.03 & 0.08 & 14.66 & 0.08 & 0.16 & 0.10 & 0.33 & 0.39 & \\
\hline Furan & 3.40 & 0.04 & 16.76 & 0.12 & 19.87 & 0.12 & 0.35 & 0.30 & 3.55 & 4.85 & \\
\hline Methanol & 1.71 & 0.01 & 11.38 & 0.04 & 16.03 & 0.06 & 0.32 & 0.20 & 3.39 & 3.68 & 2.2 \\
\hline NMA & 13.29 & 0.01 & 13.59 & 0.04 & 16.32 & 0.03 & 0.21 & 0.10 & 1.52 & 1.73 & 1.2 \\
\hline THF & 9.01 & 0.06 & 17.90 & 0.07 & 20.05 & 0.15 & 0.33 & 0.31 & 3.92 & 4.63 & \\
\hline Toluene & 11.45 & 0.09 & 17.45 & 0.07 & 19.77 & 0.11 & 0.26 & 0.23 & 2.75 & 3.00 & \\
\hline TFE & 11.21 & 0.04 & 16.54 & 0.11 & 18.75 & 0.12 & 0.20 & 0.16 & 1.32 & 1.56 & 0.6 \\
\hline \multicolumn{12}{|l|}{ Water $^{d}$} \\
\hline $\mathrm{F} 3 \mathrm{C}^{89}$ & 0.04 & 0.00 & 11.54 & 0.06 & 50.50 & 0.25 & 0.25 & 0.06 & & & \\
\hline $\mathrm{SPC} / \mathrm{E}^{90}$ & - & - & 12.03 & 0.03 & 53.05 & 0.14 & 0.29 & 0.07 & & & \\
\hline TIP4P-Ew ${ }^{91}$ & - & - & 9.53 & 0.07 & 49.79 & 0.07 & 0.24 & 0.05 & & & \\
\hline
\end{tabular}


$15 \mathrm{~cm}^{-1}$ too low, which we estimate would account for a $1-2 \mathrm{cal} \mathrm{mol}^{-1} \mathrm{~K}^{-1}(2 \%)$ increase in the total entropy.

The almost $1: 1$ partition between the translational and rotational entropies is in contrast with the case of water, where a ratio of $2: 1$ was obtained by $\mathrm{Henchman}^{44}$ and $4: 1$ from Lin et $a l .{ }^{39}$ The liquids considered here are significantly larger than water, with far weaker hydrogen bonds, in the case of the polar protic solvents. Consequently, the rotations in these systems are not as hindered as in water, which has been shown to reorganize by a jump rather than continuous mechanism. ${ }^{45-47}$ Lower frequency rotations contribute more to the total entropy than higher frequency rotations and the "solid-like" rotations (hindered-rotors) contribute at least $70 \%$ to the rotational entropy, as determined by the rotational fluidicity factor $f_{\text {rot }}$ (Table 6).

We find a large correlation ( $85 \%$ ) between the translation entropy and the self-diffusion constants, which is not surprising as the low frequency librational modes contribute most to the translational (as well as the overall) entropy. We note that the translational entropy includes components due to pure diffusion (a hard-sphere gas) as well as solid-like translations, as determined by the fluidicity parameter $f_{\text {trans }}$ (Table 6). The fraction of the translational degrees of freedom that are diffusional range from 0.16 for DMSO to 0.35 for furan, thus over $65 \%$ of the translational entropy arises from solid-like translation.

We find that $2 \mathrm{PT}$ is somewhat insensitive to the selfdiffusion constant. The self-diffusion constants for all 15 liquids are calculated over the 20 ps trajectory using the Green-Kubo (GK) approach (equation A.II.2b, ESI $\dagger$ ). This can be compared to the more common mean-squared displacement (MSD) approach, which is evaluated over the entire $2.5 \mathrm{~ns}$ MD. We find that the GK diffusion constants are not converged (as noted previously, convergence is only obtained after $\sim 100 \mathrm{ps}$ ) and are 19\% higher than the converged MSD diffusion constants. Additionally, both methods overestimate the experimental diffusion constants by $90 \%$, for the 3 of the 15 liquids for which such experimental self-diffusion constants are available. In spite of these errors, the standard molar entropies show good agreement with experiment.

To examine one specific example, consider methanol. The diffusion constant is overestimated by $112 \%$ using GK and $82 \%$ using MSD, compared to experiments. Since translations contribute $55 \%$ to the total entropy, errors due to overestimating the diffusion constant could be significant. However, the calculated entropy of methanol with OPLS $\mathrm{AA} / \mathrm{L}$ of $29.12 \mathrm{cal} \mathrm{mol}^{-1} \mathrm{~K}^{-1}$ is in excellent agreement with the experimental value of $30.40 \mathrm{cal} \mathrm{mol}^{-1} \mathrm{~K}^{-1}$. This indicates that the rotational and internal vibrational entropies are correspondingly underestimated, but only by $10 \%$, as diffusion only contributes $19 \%$ to the translational entropy.

It would thus be practical to use such 2PT calculations for entropy (the enthalpy/internal energy can also be evaluated in the same framework) to refine the forcefield against accurate experimental data at room temperature. Usually, forcefields are fitted to data at $0 \mathrm{~K}$, say from QM, leading usually to poor equilibrium densities at $300 \mathrm{~K}$. A possible improvement would be to use 2 PT to match the experimental thermodynamics and the bulk properties simultaneously. Of course, since 2PT needs only $\sim 20$ ps of $\mathrm{MD}$, it could also be used with ab initio QM MD to avoid forcefields all together. However most QM methods have difficulty in describing the London dispersion (van der Waals attraction) ${ }^{48-50}$ so important in molecular solvents.

\section{II.f Comparisons to previous methods}

There has been no comprehensive study of the entropy of the 15 pure liquids presented here using alternative methods. There has however been considerable computational effort dedicated towards calculating accurate entropies of water: the simplest liquid and most important solvent. White and Meirovitch $^{51,52}$ used a hypothetical scanning method to determine the absolute entropy and free energy and obtained excellent agreement to experiments. Lazaridis and Karplus ${ }^{53}$ later obtained standard molar entropies of water using truncated expansion of molecular pair correlation functions, ${ }^{54}$ and Sharma et al. ${ }^{55}$ using the atom-atom pair correlation function. Tyka et al. ${ }^{56}$ demonstrated the determination of absolute entropy of water using thermodynamic integration with a harmonic reference state. More recently, Henchman ${ }^{57}$ proposed a cell theory which provides reliable entropies of liquid water based on harmonic approximations.

In spite of the good agreements to experiment, it is not clear how these newer methods would be applied to systems other than water. ${ }^{39}$ Further, asymmetry in hydrogen bonding may dictate that the simple harmonic approximations of these methods break down for conditions other than the ambient ones considered. Finally, except for the Cell Method of Henchman, ${ }^{57}$ the computational cost of the aforementioned approaches would still be prohibitive for studying large biomolecular systems.

A recent study by Lin et al. ${ }^{39}$ showed that $2 \mathrm{PT}$ is accurate in predicting the absolute thermodynamics of liquid water and vapor phases along the vapor-liquid equilibrium curve, from the triple point to the critical point. 2PT achieves the same accuracy as other more common methods, while being orders of magnitude more efficient.

\section{Computational methods}

The standard molar entropy $S^{0}$, molar heat capacity $C_{p}$ and heat of vaporization $\Delta H_{\mathrm{vap}}$ are the three important observables used to characterize the thermodynamics of condensed phase systems. We have devoted most of our efforts in presenting the results of $S^{0}$ which we calculate self-consistently from the dynamics, since it is an exact quantity that does not rely on a predefined reference state. ${ }^{58-60}$ We note that $S^{0}$ is not directly accessible to methods such as umbrella sampling ${ }^{14}$ and thermodynamic integration, ${ }^{4}$ which provide the more familiar change in entropy $\Delta S .^{61}$

Since the $C_{p}$ tests the response of the entropy to small changes in temperature, we presented our predictions of $C_{p}$, directly from the DoS. This was compared to experiments and to other theoretical studies that are based on either numeric differentiation of simulated enthalpies over a range of temperatures or on Kirkwood type fluctuation analysis, ${ }^{62}$ both of which require much longer trajectories. We also calculated $\Delta H_{\text {vap }}$, though since this has been characterized for these systems in other studies, ${ }^{9,10,12,13,36,43,63}$ we do not discuss these 
results in the text. For completeness, the calculated $\Delta H_{\text {vap }}$ for all 15 liquids is presented in Table S2 (ESI $\dagger$ ).

Finally, we evaluated the various nonbonded parameters of each forcefield by calculating physical properties that are sensitive to variations therein: density, self-diffusion constant, static dielectric constant, isothermal compressibility and coefficient of thermal expansion. The methods used to obtain these measures are detailed in Appendix II.2 of ESI. $\dagger$

\section{III.a Choice of liquids and forcefields}

We classify organic liquids into three broad categories, according to their dielectric constants (Table 1), miscibility in water, and their ability to participate in hydrogen bonding:

1. Non-polar-low dielectric constant/not miscible in water e.g. benzene.

2. Polar aprotic-low dielectric constant/miscible in water/cannot hydrogen-bond (HB) e.g. acetone.

3. Polar protic - high dielectric constant/miscible in water/can HB e.g. acetic acid.

We selected 15 of the most common solvents used in organic reactions (Fig. 1) to test the accuracy of the various forcefields. Eleven of these liquids have high quality $S^{0}$ measurements from experiment. ${ }^{64}$ The remaining three (chloroform, NMA and 2,2,2triflouroethanol-TFE) were presented here as a priori predictions.

The non-polar liquids provide a rigorous test of the van der Waals (vdW) parameters, the polar protic molecules test primarily the atom centered charges and any effect due to the missing charge polarization, while the polar aprotic molecules is a good test of both and the accuracy of the HB description in the FF. We include four flexible liquids (NMA, TFE, ethylene glycol and ethanol) in our test set. Accurate determination of the thermodynamics of flexible molecules is more computationally challenging than rigid molecules, due to the need for extensive torsional sampling. The selected molecules thus provide simultaneously a rigorous test of the precision of the 2PT method and the accuracy of the forcefields.

The four forcefields in this study are commonly used for biomolecular simulations and solvation calculations, thus

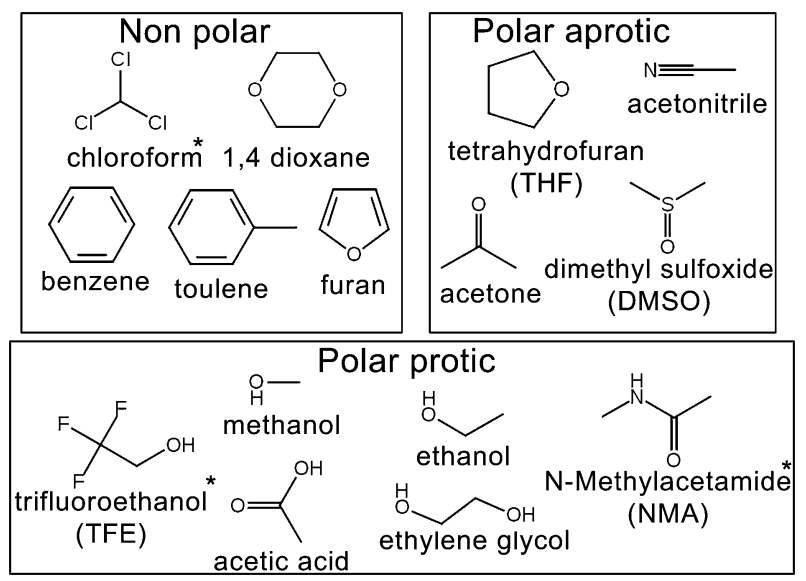

Fig. 1 The 15 organic liquids used in this study. Molecules with * symbol (TFE, chloroform and NMA) have no experimentally determined standard molar entropies and are presented here as a proiri predictions. their ability to reproduce the experimental $S^{0}$ and $C_{p}$ is of interest:

(a) AMBER 2003 $3^{30,31}$ - the AMBER99 forcefield, with the PARMBSC $0^{31}$ modifications, is a standard for molecular simulations of proteins and nucleic acids.

(b) $\mathrm{GAFF}^{32}$ - the General Amber Forcefield was created for rational drug design and simulations of small organic molecules.

(c) OPLS AA/ $\mathrm{L}^{11,33}$ - a variant of the all atom OPLS all atom forcefield, parameterized to reproduce the solvation free energies of various organic liquids from Monte Carlo simulations.

(d) Dreiding ${ }^{34}$ - a generic forcefield useful for predicting structures and dynamics of organic, biological, and maingroup inorganic molecules.

Dreiding is the simplest of the forcefields considered here, with just seven parameters to describe all valence interactions. Dreiding is also the only forcefield with an explicit three-body hydrogen bonding term (see Appendix I (ESI $\dagger$ ) for the description of the forcefields).

\section{III.b Liquid simulations}

All simulations were performed using the LAMMPS ${ }^{65,66}$ simulation engine, which affords the flexibility of using various forcefields in a common framework (we modified LAMMPS to include the full Dreiding FF, including 3-body HB). Long-range coulombic interactions were calculated using the particle-particle particle-mesh Ewald method ${ }^{67}$ (with a precision of $10^{-5} \mathrm{kcal} \mathrm{mol}^{-1}$ ), while the van der Waals interaction was computed with a cubic spline (an inner cutoff of $11 \AA$ and an outer cutoff of $12 \AA$ ). We used the spline to guarantee that the energies and forces go smoothly to zero at the outer cutoff, preventing energy drifts that might arise from inconsistent forces. We also tested the effect of the cutoff by computing the energy of benzene with cutoffs ranging from 8 to $20 \AA$ and found converged results at $12 \AA$.

For each system, we used the Continuous Configurational Boltzmann Biased (CCBB) Monte Carlo (MC) method ${ }^{68,69}$ to generate a random starting structure of 512 solvent molecules packed to minimize the system interaction energy. To rapidly equilibrate the systems, we used our standard procedure: $:^{70-72}$ after an initial conjugant gradient minimization to an RMS force of $10^{-4} \mathrm{kcal} \mathrm{mol}^{-1} \AA^{-1}$, the system was slowly heated from $0 \mathrm{~K}$ to $298 \mathrm{~K}$ over a period of 100 ps using a Langevin thermostat in the constant temperature, constant volume canonical (NVT) ensemble. The temperature coupling constant was $0.1 \mathrm{ps}$ and the simulation timestep was $1.0 \mathrm{fs}$.

This equilibration was followed by 1 ns of constant-pressure(iso-baric), constant-temperature (NPT) dynamics at $298 \mathrm{~K}$ and $1 \mathrm{~atm}$. The temperature coupling constant was $0.1 \mathrm{ps}$ while the pressure piston constant was 2.0 ps. The equations of motion used are those of Shinoda et al. ${ }^{73}$ which combine the hydrostatic equations of Martyna et al. ${ }^{74}$ with the strain energy proposed by Parrinello and Rahman. ${ }^{75}$ The time integration schemes closely follow the time-reversible measure-preserving Verlet integrators derived by Tuckerman et al. ${ }^{76}$ Production dynamics was then run for a further $2.5 \mathrm{~ns}$ in the NPT ensemble, with coordinates and velocities saved every $4 \mathrm{ps}$ for post-trajectory analysis. 

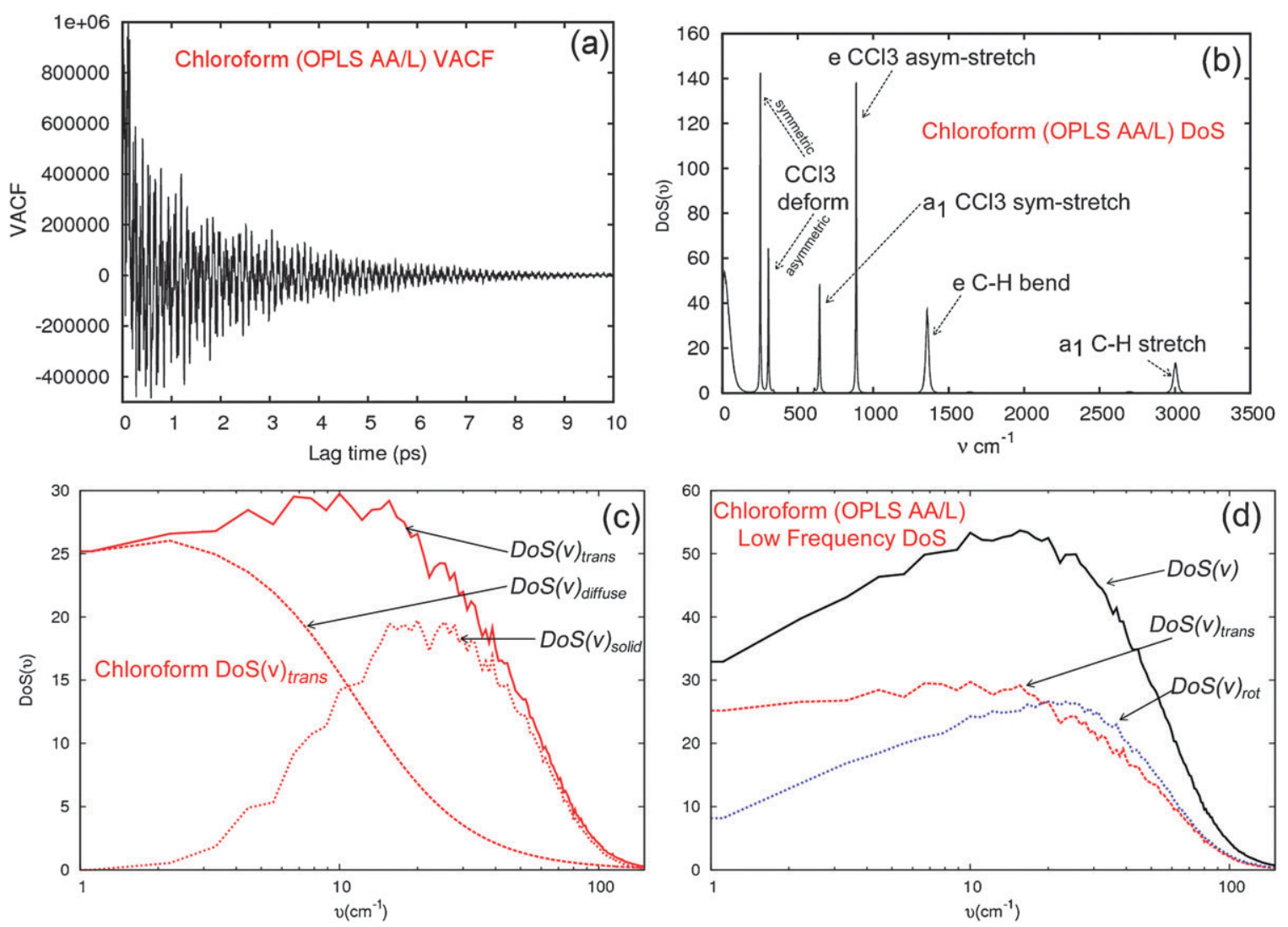

Fig. 2 (a) Velocity autocorrelation function (VACF) of chloroform from 20 ps NVT MD, using the OPLS AA/L forcefield. (b) The corresponding density of states (DoS), obtained from the Fourier Transform of the VACF. The 6 valence infrared and Raman active modes $\left(\mathrm{a}_{1}: 3001 \mathrm{~cm}{ }^{-1} \mathrm{C}-\mathrm{H}\right.$ stretch, $634 \mathrm{~cm}^{-1} \mathrm{CCl} 3$ symmetric stretch and $301 \mathrm{~cm}^{-1} \mathrm{CCl} 3$ symmetric deform. e: $1355 \mathrm{~cm}^{-1} \mathrm{CH}$ bend, $875 \mathrm{~cm}^{-1} \mathrm{CCl} 3$ asymmetric stretch and $239 \mathrm{~cm}^{-1} \mathrm{CCl} 3$ asymmetric deform) are labeled. These can be compared to 3034, 680, 363, 1220, 774 and 261 from experiment. ${ }^{95}$ (c) The $2 \mathrm{PT}$ partitioning of the translational component of the DoS into the solid $\left(\mathrm{DoS}_{\text {solid }}\right)$ and gas $\left(\operatorname{DoS}_{\mathrm{diffuse}}\right)$ components. Note that $\operatorname{DoS} \mathrm{S}_{\mathrm{vib}}=0$ at $v=0$, while the gas component has value $\operatorname{DoS}(0)=32.4$ at $v=0$ and smoothly decays to 0 over $150 \mathrm{~cm}^{-1}$. The fraction of the modes in the gas phase (the $f$ - fludicity - factor), and hence the rate of decay of $\mathrm{DoS}_{\text {diffuse, }}$, is determined self-consistently from the diffusivity of the systems $(f=0.32$ in this system). The $f$ factor and $\operatorname{DoS}(0)$ parameters of the $2 \mathrm{PT}$ method are extracted directly from the MD trajectory. (d) The low frequency librational modes $\left(0-250 \mathrm{~cm}^{-1}\right)$ including the translational $\mathrm{DoS}_{\text {trans }}$ and rotational $\operatorname{DoS}_{\text {rot }}$ components (log scale). Here, the value of the DoS at $v=0$ $[\operatorname{DoS}(0)]=32.4$, which would lead to an infinite contribution to the entropy if the harmonic approximation was employed. The thermodynamic properties of the system are obtained by applying the $2 \mathrm{PT}$ correction and integrating over the DoS with the appropriate weighting functions.

\section{III.c Obtaining thermodynamic properties: the 2PT method}

The 2PT-MD method uses the following protocol to obtain free energies from the $2.5 \mathrm{~ns}$ of NPT MD: we analyzed the trajectory in 500 ps blocks and selected the snapshot with volume closest to the average value. Then using the coordinates and dynamics from this snapshot we ran a short 20 ps NVT MD, saving the coordinates and velocities every $4 \mathrm{fs}$ (needs to be shorter than the fastest vibrational levels which have periods of $\sim 10$ fs for a $3000 \mathrm{~cm}^{-1}$ vibration), for a total of 5000 structures. The quoted thermodynamic values are taken as the statistical average over these 5 trajectories.

For each 20 ps trajectory, we calculated the velocity autocorrelation function (VAC) for each atom,

$$
C(t)=\sum_{j=1}^{N} \sum_{k=1}^{3} m_{j}\left[\lim _{t \rightarrow \infty} \frac{1}{2 \tau} \int_{-\tau}^{\tau} v_{j}^{k}\left(t^{\prime}+t\right) v_{j}^{k}\left(t^{\prime}\right) \mathrm{d} t^{\prime}\right]
$$

where $m_{j}$ is the mass of atom $j ; v_{j}^{k}(i)$ the $k$-th component of the velocity of atom $j$ at time $t$.
The total density of states (DoS) DoS(v) (also referred to as the power spectrum or spectral density) is obtained from a fast Fourier Transform (FFT) of eqn (2) (Fig. 2):

$$
\operatorname{DoS}(v)=\lim _{t \rightarrow \infty} \frac{1}{2 k T} \int_{-\tau}^{\tau} C(t) e^{-2 \pi v t} \mathrm{~d} t
$$

Physically, $\operatorname{DoS}(v)$ represent the density of normal modes of the system at frequency $v$. This total DoS is then partitioned into $\operatorname{DoS}(v)=\operatorname{DoS}(v, f)_{\text {diff }}+\operatorname{DoS}(v)_{\text {solid. }}{ }^{24}$

III.c.i The diffusive component $\operatorname{DoS}(v)_{\text {diff }}$ The diffusive component $\operatorname{DoS}(v)_{\text {diff }}$ is described as a gas of hard spheres:

$$
\operatorname{DoS}(v, f)_{\text {diff }}=\frac{\operatorname{DoS}(0)}{1+\left[\frac{\operatorname{DoS}(0) \pi v}{6 f N}\right]^{2}}
$$

in terms of

(1) the total $\operatorname{DoS}(0)$ at $v=0$, this is,

$$
\operatorname{DoS}(0)=\operatorname{DoS}(0)_{\text {diff }}
$$




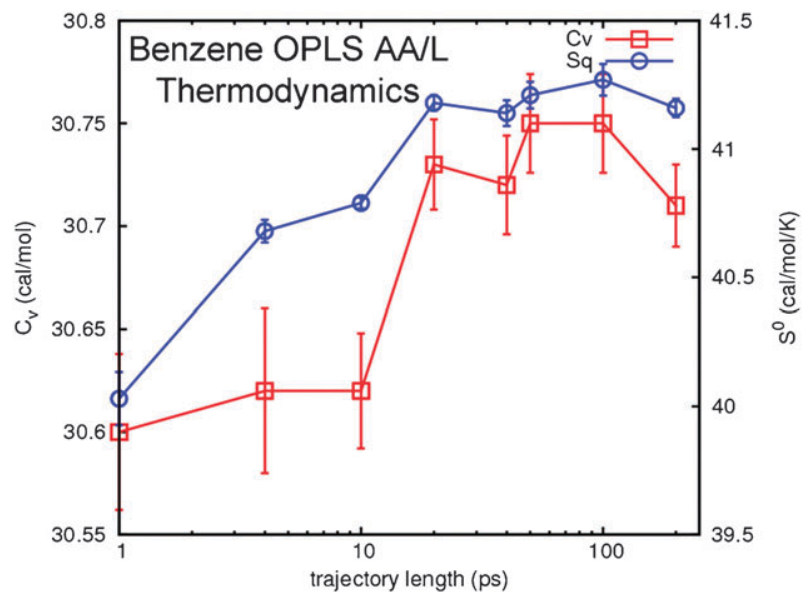

Fig. 3 The molar heat capacity $C_{v}$ (squares) and standard molar entropy $S^{0}$ (circles) of benzene using the OPLS AA/L forcefield. The left $y$-axis is the $C_{v}$, the right $y$-axis is the $S^{0}$ and the $x$-axis is the log scale of the trajectory length. Convergence in both thermodynamic quantities is observed from 20 ps trajectories. The uncertainty in the measurements $\left[0.14 \mathrm{cal} \mathrm{mol}^{-1}(0.6 \%)\right.$ for $C_{v}$ and $0.25 \mathrm{cal} \mathrm{mol}^{-1} \mathrm{~K}^{-1}$ $(0.7 \%)$ for $\left.S^{0}\right]$ is obtained from 5 independent trajectories and scale by a factor of 5 for presentation purposes.

[Note that for translational modes $\operatorname{DoS}(0)$ is related to the self-diffusivity coefficient $D$ as $\operatorname{DoS}(0)=\frac{12 m N D}{k T}$, where $N$ is the number of molecules in the system and $m$ is the mass of a molecule], and

(2) the "fluidicity" parameter $f$, which is the fraction of the $3 N$ translation or rotation modes corresponding to the fluid or diffusional parts of the dynamic system, i.e.

$$
\int_{0}^{\infty} \operatorname{DoS}(v, f)_{\mathrm{diff}} \mathrm{d} v=3 f N
$$

$f$ is obtained from the normalized self-diffusivity $(\Delta)$ of the system: ${ }^{24}$

$$
\begin{gathered}
2 \Delta^{-9 / 2} f^{15 / 2}-6 \Delta^{-3} f^{5}-\Delta^{-3 / 2} f^{7 / 2}+6 \Delta^{-3 / 2} f^{5 / 2}+2 f-2 \\
=0
\end{gathered}
$$

where the value of $\Delta$ is determined from the state variables of the fluid $^{24}$

$$
\Delta(T, V, N, m, \operatorname{DoS}(0))=\frac{2 \operatorname{DoS}(0)}{9 N}\left(\frac{\pi k T}{m}\right)^{1 / 3}\left(\frac{N}{V}\right)^{1 / 3}\left(\frac{6}{\pi}\right)^{2 / 3}
$$

$\operatorname{DoS}(v)_{\text {diff }}$ contains all effects due to anharmonicity and diffusion in the system, which are most important in the low frequency regime.

III.c.ii The solid component DoS(v) solid. For the solid component $\operatorname{DoS}(v)_{\text {solid, }}$ each vibrational mode can be considered as harmonic and one can write the canonical partition function $Q$ (from which all thermodynamic quantities are calculated) as:

$$
\ln Q=\int_{0}^{\infty} \operatorname{DoS}(v)_{\mathrm{solid}} q_{\mathrm{HO}}(v) \mathrm{d} v
$$

where $q_{\mathrm{HO}}(v)=\frac{\exp (-\beta h v)}{1-\exp (-\beta h v)}$ is the quantum harmonic oscillator partition function, $\beta=1 / k T$ and $h$ the Planck's constant.

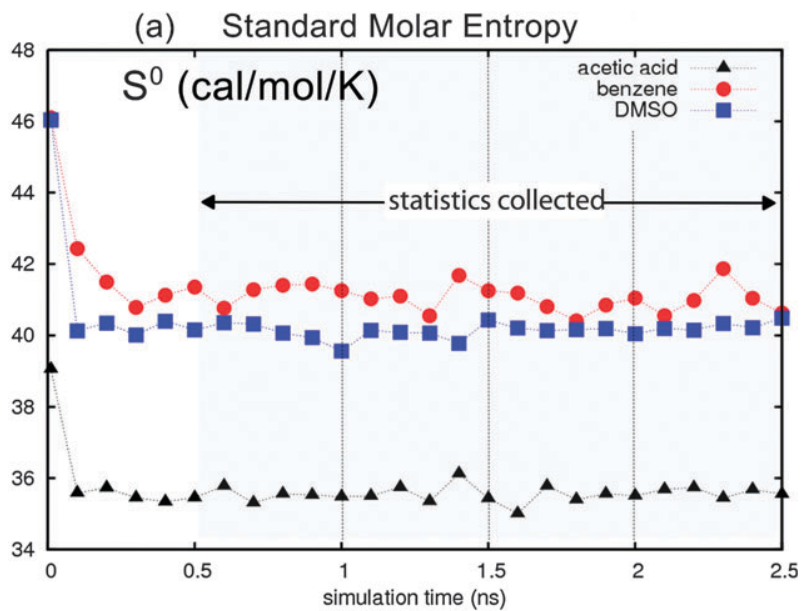

(b) Specific heat capacity

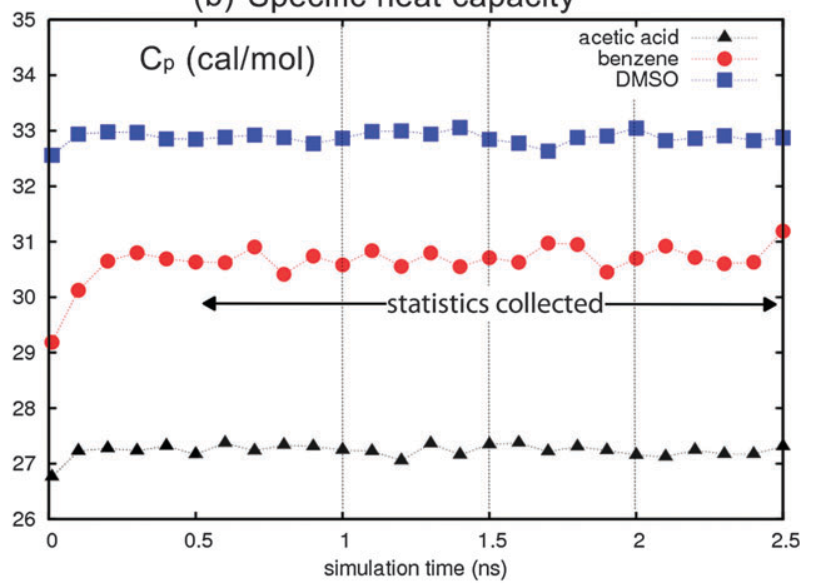

Fig. 4 (a) Standard molar entropy $S^{0}$ for acetic acid (triangles), benzene (circles) and DMSO (squares) using the OPLS AA-L forcefield, evaluated every 100 ps during $2.5 \mathrm{~ns}$ dynamics. (b) Molar heat capacity $C_{p}$ convergence is observed after $500 \mathrm{ps}$, validating the simulation protocol. Statistics are obtained every 500 ps after equilibration; the average calculated from the 5 discrete points is within $0.1 \%$ of the running average calculated every $100 \mathrm{ps}$. We find average fluctuations in $S^{0}$ of $0.36 \mathrm{cal} \mathrm{mol}^{-1} \mathrm{~K}^{-1}(0.9 \%)$ and in $C_{p}$ of $0.12 \mathrm{cal} \mathrm{mol}^{-1} \mathrm{~K}^{-1}(0.6 \%)$.

$S^{0}$ and $C_{v}$ are then obtained directly from the standard statistical mechanical expressions:

$$
\begin{gathered}
S^{0}=k \ln Q+\beta^{-1}\left(\frac{\partial \ln Q}{\partial T}\right)_{N, V} \\
=k \int_{0}^{\infty} \operatorname{DoS}(v)_{\text {solid }} W_{\text {solid }}^{S}(v) \mathrm{d} v \\
C_{v}=\left(\frac{\partial S^{0}}{\partial T}\right)_{N, V}=k\left(\frac{\partial \ln Q}{\partial T}\right)_{N, V}+\beta^{-1}\left(\frac{\partial^{2} \ln Q}{\partial T^{2}}\right)_{N, V} \\
=k^{2} \beta^{-2} \int_{0}^{\infty} \operatorname{DoS}(v)_{\text {solid }} W_{\text {solid }}^{C_{v}}(v) \mathrm{d} v
\end{gathered}
$$

with weighting functions

$$
\begin{aligned}
& W_{\text {solid }}^{S}(v)=\frac{\beta h v}{\exp (\beta h v)-1}-\ln [1-\exp (-\beta h v)] \\
& W_{\text {solid }}^{C_{v}}(v)=\frac{\exp (-\beta h v)}{[1-\exp (-\beta h v)]^{2}}
\end{aligned}
$$




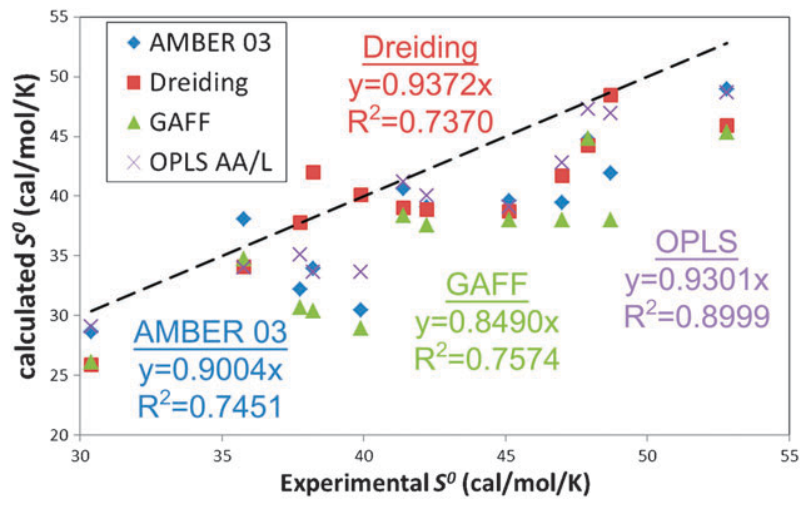

Fig. 5 Comparison of experimental and calculated standard molar entropies $S^{0}\left(\mathrm{cal} \mathrm{mol}^{-1} \mathrm{~K}^{-1}\right)$ for 12 of the 15 liquids in this study. No experimental data are available for chloroform, NMA and TFE; the calculated values of $43.01,40.23$ and $43.54 \mathrm{cal} \mathrm{mol}^{-1} \mathrm{~K}^{-1}$, respectively, are presented as a prori predictions. The precision in the calculated values is $\sim 0.25 \mathrm{cal} \mathrm{mol}^{-1} \mathrm{~K}^{-1}$. The dashed line indicates exact matching between simulation and experiment. All four of the forcefields underestimate $S^{0}$. The OPLS AA/L forcefield provides the best performance with a $90 \%$ correlation. The generic Dreiding forcefield $(74 \%)$ is as accurate as the AMBER class of forcefields.

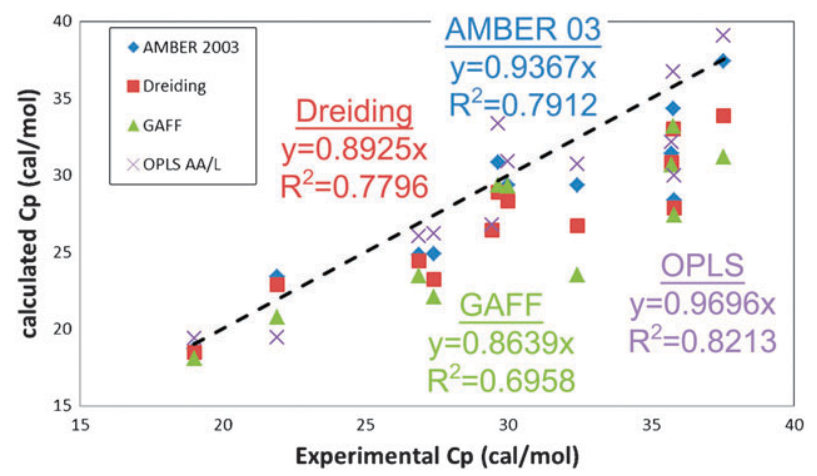

Fig. 6 Comparison of constant pressure heat capacity $C_{p}\left(\mathrm{cal} \mathrm{mol}^{-1}\right)$ for the 12 liquids with experimental data. The dashed black line indicates exact matching between simulation and experiment. The $C_{p}$ is obtained from the calculated $C_{v}$ according to eqn (1) (see Table $\mathrm{S} 4, \mathrm{ESI} \dagger)$. The OPLS AA/L forcefield provides the best agreement with experiment, with a correlation coefficient of $82 \%$, while the GAFF forcefield has the worse agreement (70\%).

From (5) we see that $\operatorname{DoS}(v)_{\text {solid }} \rightarrow 0$ as $v \rightarrow 0$, so that no singularities occur from using the harmonic oscillator partition function for $\operatorname{DoS}(v)_{\text {solid }}$.

The total standard molar entropy and heat capacity are then obtained as:

$$
\begin{gathered}
S^{0}=k \int_{0}^{\infty}\left[\operatorname{DoS}(v)_{\mathrm{diff}} W_{\mathrm{diff}}^{S}(v)+\operatorname{DoS}(v)_{\mathrm{solid}} W_{\mathrm{solid}}^{S}(v)\right] \mathrm{d} v \\
C_{v}=k \int\left[\operatorname{DoS}(v)_{\mathrm{diff}} W_{\mathrm{diff}}^{C_{v}}(v)\right. \\
\left.+\operatorname{DoS}(v)_{\text {solid }} W_{\text {solid }}^{C_{v}}(v)\right] \mathrm{d} v
\end{gathered}
$$

where

$$
\begin{aligned}
& W_{\mathrm{diff}}^{S}=W_{\mathrm{HS}}^{S}(v)=\frac{1}{3} \frac{S^{\mathrm{HS}}}{k} \\
& W_{\mathrm{diff}}^{C_{v}}=W_{\mathrm{HS}}^{C_{v}}(v)=0.5
\end{aligned}
$$

are the weighting functions of a Carnahan-Starling ${ }^{77}$ hard sphere gas with entropy $S^{\mathrm{HS}}$.

III.c.iii Application to molecular systems. 2PT relies only on the trajectory of individual atomic velocity vectors, allowing logical groups in the system to be grouped together to compute their thermodynamics consistently and independently. Decomposition of the velocity vector for molecules can be achieved by considering the translational (diffusional) $S_{\text {trans }}$, rotational $S_{\text {rot }}$ and internal vibrational components $S_{\text {vib: }}$ :

- $S_{\text {trans: }}$ the center of mass translational contribution to the total velocity ( $V_{\text {trans }}$ ) (for molecule $i$ and total mass $M_{i}$ ) is obtained as the center of mass velocity of that molecule:

$$
V_{\text {trans }}(i)=\frac{1}{M_{i}} \sum_{k=1}^{3} \sum_{j} m_{j} V_{j}^{k}
$$

where $k$ is the $k^{\prime}$ th component of the velocity vector of atom $j$ in molecule $i$. The translational entropy is obtained by substituting $V_{\text {trans }}$ into eqn (2). The translational DoS $\left[\operatorname{DoS}_{\text {trans }}(v)\right]$ can then be decomposed into the diffusional and solid-like components according to the $f_{\text {trans }}$ fluidicity factor as defined in eqn (7).

- $S_{\text {rot }}$ : the rotational contribution $\left(V_{\text {rot }}\right)$ is obtained by calculating the angular velocity ( $\left.V_{\text {ang }}\right)$, treating the system as a quantum rigid rotor:

$$
\begin{gathered}
V_{\mathrm{rot}}(i)=\omega(i) \times V_{\mathrm{tot}}(i) \\
\omega(i)=I_{i}^{-1} \times L(i)
\end{gathered}
$$

where $I_{i}^{-1}$ is the inverse of the moment of inertial tensor for molecule $i$ and $L(i)$ is the angular momentum:

$$
L(i)=\sum_{j} m_{j}\left(R_{j} \times V_{j}\right)
$$

(here $R_{j}$ is the position of atom $j$ in molecule $i$ )

The rotational entropy is obtained by substituting $V_{\text {ang }}$ into eqn (2), with weighting functions:

$$
\begin{aligned}
& W_{\text {rot }}^{S}(v)=\frac{1}{3} \frac{S^{\mathrm{R}}}{k} \\
& W_{\operatorname{rot}}^{C_{v}}(v)=0.5
\end{aligned}
$$

where $S^{\mathrm{R}}$ is the rotational entropy of the molecule in the ideal gas state (free rigid rotor). Analogous to the $\operatorname{DoS}_{\text {trans }}(v)$, the $\operatorname{DoS}_{\text {rot }}(v)$ can be decomposed into the diffusional and solid components, with rotational fluidicity factor $f_{\text {rot }}$ obtained from eqn (7).

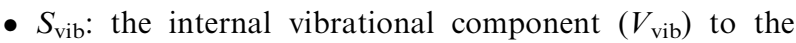
velocity is taken as the remaining velocity after subtracting the first two contributions: $V_{\text {vib }}(i)=V_{\text {tot }}(i)-\left[V_{\text {trans }}(i)+V_{\text {rot }}(i)\right]$. The vibrational entropy is obtained by substituting $V_{\text {vib }}$ into eqn (2). There is no decomposition of the DoS here, as $\operatorname{DoS}_{\mathrm{vib}}(v)$ has no diffusional component (i.e. the fluidicity is zero).

\section{Conclusions}

We have characterized the thermodynamics of 15 pure organic liquids using the 2PT method. Good agreement with experiment is obtained in the calculated standard molar 
entropies and excellent agreement is obtained for the molar heat capacities with all four common empirical forcefields. Overall, the highly optimized OPLS AA/L forcefield is the most accurate for obtaining thermodynamics of these liquids.

We partitioned the molar entropies into the contributions arising from translation, rotation and internal vibration, and find that a non-negligible $17 \%$ of the entropy arises from intra-molecular vibrations, possibly indicating the need for future forcefields to be better tuned to reproduce experimental vibrational frequencies.

Thus 2PT offers a consistent, parameter free method for accurately determining the standard molar entropy and heat capacity of arbitrary liquids, with a high thermodynamic precision. Due to its efficiency (adding $\sim 0.2 \%$ to the total simulation time), we foresee future uses in obtaining entropies of more complex, condensed phased systems.

\section{Acknowledgements}

The authors acknowledge Mario Blanco, and Prabal Maiti for useful discussions. This project was partially supported by grants to Caltech from National Science Foundation (CMMI-072870, CTS-0608889). This work is supported by the WCU program (31-2008-000-10055-0) through the National Research Foundation of Korea and the generous allocation of computing time from the KISTI supercomputing center. TAP thanks the US Department of Energy CSGF and the National Science Foundation for graduate fellowships. Prof. Goddard acknowledges the WCU program at KAIST for financial support.

\section{References}

1 B. Marten, K. Kim, C. Cortis, R. A. Friesner, R. B. Murphy, M. N. Ringnalda, D. Sitkoff and B. Honig, J. Phys. Chem., 1996, 100, 11775-11788.

2 D. J. Tannor, B. Marten, R. Murphy, R. A. Friesner, D. Sitkoff, A. Nicholls, M. Ringnalda, W. A. Goddard and B. Honig, J. Am. Chem. Soc., 1994, 116, 11875-11882.

3 F. J. Webster, J. Schnitker, M. S. Friedrichs, R. A. Friesner and P. J. Rossky, Phys. Rev. Lett., 1991, 66, 3172-3175.

4 X. Kong and C. L. Brooks Iii, J. Chem. Phys., 1996, 105, 2414-2423.

5 J. G. Kirkwood, J. Chem. Phys., 1935, 3, 300-313.

6 R. Zwanzig, J. Chem. Phys., 1954, 22, 1420-1426.

7 E. Darve and A. Pohorille, J. Chem. Phys., 2001, 115, 9169-9183.

8 B. Widom, J. Chem. Phys., 1963, 39, 2808-2812.

9 J. M. Briggs, T. B. Nguyen and W. L. Jorgensen, J. Phys. Chem., 1991, 95, 3315-3322.

10 W. L. Jorgensen and D. L. Severance, J. Am. Chem. Soc., 1990, 112, 4768-4774

11 W. L. Jorgensen and J. Tiradorives, J. Am. Chem. Soc., 1988, 110, 1657-1666.

12 W. L. Jorgensen and J. M. Briggs, Mol. Phys., 1988, 63, 547-558.

13 W. L. Jorgensen, J. Phys. Chem., 1986, 90, 1276-1284.

14 G. M. Torrie and J. P. Valleau, J. Comput. Phys., 1977, 23, 187-199.

15 G. D. Rose and R. Wolfenden, Annu. Rev. Biophys. Biomol. Struct., 1993, 22, 381-415.

16 P. L. Privalov and G. I. Makhatadze, J. Mol. Biol., 1993, 232, 660-679.

17 E. M. Boczko and C. L. Brooks, Science, 1995, 269, 393-396.

18 R. S. Spolar and M. T. Record, Science, 1994, 263, 777-784.

19 D. M. Huang and D. Chandler, J. Phys. Chem. B, 2002, 106, 2047-2053.
20 G. Hummer, S. Garde, A. E. Garcia, M. E. Paulaitis and L. R. Pratt, J. Phys. Chem. B, 1998, 102, 10469-10482.

21 B. Guillot, Y. Guissani and S. Bratos, J. Chem. Phys., 1991, 95, $3643-3648$.

22 T. Lazaridis and M. E. Paulaitis, J. Phys. Chem., 1992, 96, 3847-3855.

23 B. Lee, Biopolymers, 1991, 31, 993-1008.

24 S. T. Lin, M. Blanco and W. A. Goddard, J. Chem. Phys., 2003, 119, 11792-11805.

25 S. T. Lin, P. K. Maiti and W. A. Goddard, J. Phys. Chem. B, 2005, 109, 8663-8672.

26 B. Jana, S. Pal, P. K. Maiti, S. T. Lin, J. T. Hynes and B. Bagchi, J. Phys. Chem. B, 2006, 110, 19611-19618.

27 T. A. Pascal, R. Abrol, R. Mittal, Y. Wang, N. V. Prasadarao and W. A. Goddard, J. Biol. Chem., 2010, DOI: 10.1074/ jbc.M110.122804.

28 Y. Y. Li, S. T. Lin and W. A. Goddard, J. Am. Chem. Soc., 2004, 126, $1872-1885$.

29 S. S. Jang, S. T. Lin, P. K. Maiti, M. Blanco, W. A. Goddard, P. Shuler and Y. C. Tang, J. Phys. Chem. B, 2004, 108, 12130-12140.

30 D. A. Case, T. E. Cheatham, T. Darden, H. Gohlke, R. Luo, K. M. Merz, A. Onufriev, C. Simmerling, B. Wang and R. J. Woods, J. Comput. Chem., 2005, 26, 1668-1688.

31 A. Perez, I. Marchan, D. Svozil, J. Sponer, T. E. Cheatham, C. A. Laughton and M. Orozco, Biophys. J., 2007, 92, 3817-3829.

32 J. M. Wang, R. M. Wolf, J. W. Caldwell, P. A. Kollman and D. A. Case, J. Comput. Chem., 2004, 25, 1157-1174.

33 G. A. Kaminski, R. A. Friesner, J. Tirado-Rives and W. L. Jorgensen, J. Phys. Chem. B, 2001, 105, 6474-6487.

34 S. L. Mayo, B. D. Olafson and W. A. Goddard, J. Phys. Chem., 1990, 94, 8897-8909.

35 A. K. Rappe and W. A. Goddard, J. Phys. Chem., 1991, 95, $3358-3363$.

36 N. A. McDonald and W. L. Jorgensen, J. Phys. Chem. B, 1998, 102, 8049-8059.

37 T. Fox and P. A. Kollman, J. Phys. Chem. B, 1998, 102, 8070-8079.

38 V. M. Anisimov, I. V. Vorobyov, B. Roux and A. D. MacKerell, J. Chem. Theory Comput., 2007, 3, 1927-1946.

39 S. T. Lin, P. K. Maiti and W. A. Goddard, J. Phys. Chem. B, 2010, 114, 8191-8198.

40 J. P. Ryckaert, G. Ciccotti and H. J. C. Berendsen, J. Comput. Phys., 1977, 23, 327-341.

41 M. R. Shirts and V. S. Pande, J. Chem. Phys., 2005, 122, 134508-134513.

42 T. Yan, C. J. Burnham, M. G. Del Popolo and G. A. Voth, J. Phys. Chem. B, 2004, 108, 11877-11881.

43 W. L. Jorgensen, D. S. Maxwell and J. Tirado-Rives, J. Am. Chem. Soc., 1996, 118, 11225-11236.

44 R. H. Henchman, J. Chem. Phys., 2007, 126, 064504.

45 D. Laage and J. T. Hynes, Science, 2006, 311, 832-835.

46 B. Mukherjee, P. K. Maiti, C. Dasgupta and A. K. Sood, J. Phys. Chem. B, 2009, 113, 10322-10330.

47 V. Molinero, T. Cagin and W. A. Goddard, J. Phys. Chem. A, 2004, 108, 3699-3712.

48 Y. Zhang, X. Xu and W. A. Goddard, Proc. Natl. Acad. Sci. U. S. A., 2009, 106, 4963-4968.

49 C. D. Sherrill, B. G. Sumpter, M. O. Sinnokrot, M. S. Marshall, E. G. Hohenstein, R. C. Walker and I. R. Gould, J. Comput. Chem., 2009, 30, 2187-2193.

50 C. D. Sherrill, T. Takatani and E. G. Hohenstein, J. Phys. Chem. A, 2009, 113, 10146-10159.

51 R. P. White and H. Meirovitch, Proc. Natl. Acad. Sci. U. S. A., 2004, 101, 9235-9240.

52 R. P. White and H. Meirovitch, J. Chem. Phys., 2004, 121, 10889-10904.

53 T. Lazaridis and M. Karplus, J. Chem. Phys., 1996, 105, 4294-4316.

54 L. Wang, R. Abel, R. A. Friesner and B. J. Berne, J. Chem. Theory Comput., 2009, 5, 1462-1473.

55 R. Sharma, M. Agarwal and C. Chakravarty, Mol. Phys., 2008, 106, 1925-1938.

56 M. D. Tyka, R. B. Sessions and A. R. Clarke, J. Phys. Chem. B, 2007, 111, 9571-9580. 
57 R. H. Henchman, J. Chem. Phys., 2007, 126, 064504.

58 G. S. Parks, W. D. Kennedy, R. R. Gates, J. R. Mosley, G. E. Moore and M. L. Renquist, J. Am. Chem. Soc., 1956, 78, 56-59.

59 G. S. Parks, H. M. Huffman and M. Barmore, J. Am. Chem. Soc., 1933, 55, 2733-2740.

60 G. S. Parks, H. M. Huffman and S. B. Thomas, J. Am. Chem. Soc., 1930, 52, 1032-1041.

61 Y. Marcus, Pure Appl. Chem., 1987, 59, 1093-1101.

62 H. W. Horn, W. C. Swope, J. W. Pitera, J. D. Madura, T. J. Dick, G. L. Hura and T. Head-Gordon, J. Chem. Phys., 2004, 120, 9665-9678.

63 G. Kaminski and W. L. Jorgensen, J. Phys. Chem., 1996, 100, 18010-18013.

64 NIST, Chemistry WebBook, National Institute of Standards and Technology, Reference Database Number 69 edn, 2000.

65 S. J. Plimpton, R. Pollock and M. Stevens, Proceedings of the Eighth SIAM Conference on Parallel Processing for Scientific Computing, Minneapolis, MN, 1997.

66 S. Plimpton, J. Comput. Phys., 1995, 117, 1-19.

67 R. W. Hockney and J. W. Eastwood, Computer Simulation Using Particles, Taylor \& Francis, New York, 1989.

68 P. K. Maiti, T. Cagin, G. Wang and W. A. Goddard, Macromolecules, 2004, 37, 6236-6254.

69 T. Cagin, G. F. Wang, R. Martin, N. Breen and W. A. Goddard, Nanotechnology, 2000, 11, 77-84.

70 P. K. P. Maiti, A. T. Pascal, N. Vaidehi and W. A. Goddard, J. Nanosci. Nanotechnol., 2007, 7, 1712-1720.

71 P. K. Maiti, T. A. Pascal, N. Vaidehi, J. Heo and W. A. Goddard, Biophys. J., 2006, 90, 1463-1479.

72 P. K. Maiti, T. A. Pascal, N. Vaidehi and W. A. Goddard, Nucleic Acids Res., 2004, 32, 6047-6056.

73 W. Shinoda, M. Shiga and M. Mikami, Phys. Rev. B: Condens. Matter Mater. Phys., 2004, 69, 134103.

74 G. J. Martyna, D. J. Tobias and M. L. Klein, J. Chem. Phys., 1994, 101, 4177-4189.

75 M. Parrinello and A. Rahman, J. Appl. Phys., 1981, 52, 7182-7190.
76 M. E. Tuckerman, J. Alejandre, R. Lopez-Rendon, A. L. Jochim and G. J. Martyna, J. Phys. A: Math. Gen., 2006, 39, 5629-5651.

77 N. F. Carnahan and K. E. Starling, J. Chem. Phys., 1970, 53, 600-603.

78 J. M. Wang, W. Wang, P. A. Kollman and D.A. Case, J. Mol. Graphics Modell., 2006, 25, 247-260.

79 Jaguar, version 7.0, Schrödinger, New York, NY, 2007.

80 R. S. Mulliken, J. Chem. Phys., 1955, 23, 1833-1840.

81 MacroModel, version 9.7, Schrödinger, New York, NY, 2009.

82 A. L. Macclellan, Tables of experimental dipole moments, Rahara Enterprises, El Cerrito, California, 1974

83 W. C. Swope, H. C. Andersen, P. H. Berens and K. R. Wilson, J. Chem. Phys., 1982, 76, 637-649.

84 R. Shaw, J. Chem. Eng. Data, 1969, 14, 461-465.

85 P. Brocos, E. Calvo, R. Bravo, M. Pintos, A. Amigo, A. H. Roux and G. Roux-Desgranges, J. Chem. Eng. Data, 1999, 44, 67-72.

86 E. Calvo, P. Brocos, R. Bravo, M. Pintos, A. Amigo, A. H. Roux and G. Roux-Desgranges, J. Chem. Eng. Data, 1998, 43, 105-111.

87 F. Comelli, R. Francesconi, A. Bigi and K. Rubini, J. Chem. Eng. Data, 2006, 51, 665-670.

88 J. Gao, D. Habibollazadeh and L. Shao, J. Phys. Chem., 1995, 99, 16460-16467.

89 M. Levitt, M. Hirshberg, R. Sharon, K. E. Laidig and V. Daggett, J. Phys. Chem. B, 1997, 101, 5051-5061.

90 H. J. C. Berendsen, J. R. Grigera and T. P. Straatsma, J. Phys. Chem., 1987, 91, 6269-6271.

91 H. W. Horn, W. C. Swope, J. W. Pitera, J. D. Madura, T. J. Dick, G. L. Hura and T. Head-Gordon, J. Chem. Phys., 2004, 120, 9665-9678.

92 K. R. Harris, P. J. Newitt and Z. J. Derlacki, J. Chem. Soc., Faraday Trans., 1998, 94, 1963-1970.

93 K. Krynicki, C. D. Green and D. W. Sawyer, Faraday Discuss. Chem. Soc., 1978, 66, 199-208.

94 K. J. Tauer and W. N. Lipscomb, Acta Crystallogr., 1952, 5, 606-612.

95 T. Shimanouchi, Tables of Molecular Vibrational Frequencies Consolidated Volume I, National Bureau of Standards, 1972, pp. $1-160$ 